\title{
Increasing Power Efficiency in Transmitter Diversity Systems under Error Performance Constraints
}

\author{
Diomidis S. Michalopoulos, Athanasios S. Lioumpas and George K. Karagiannidis \\ Department of Electrical and Computer Engineering \\ Aristotle University of Thessaloniki, Thessaloniki, Greece \\ Email: \{dmixalo, alioumpa, geokarag\}@auth.gr
}

\begin{abstract}
Motivated by the well-known Knapsack problem, we propose an efficient algorithmic method that minimizes and optimally allocates the total transmitting power in transmitter diversity systems, provided that the instantaneous Bit-ErrorRate (BER) is not greater than a predetermined value. We also provide closed-form expressions for the average total transmitted power for the case of two transmitting antennas operating in Rayleigh fading, and the average number of active antennas at the transmitter assuming Nakagami- $m$ fading channels. Simulations and numerical results show that, compared to the conventional equi-power scheme, the proposed model offers a considerable reduction in the total transmitting power and the average number of active antennas, without loss in error performance.
\end{abstract}

\section{INTRODUCTION}

Transmitter diversity provides diversity gain in cases when multiple transmitting antennas and a single antenna at the receiver are employed [1]-[3]. Its operation is based upon the separation of the transmitting signals using Code Division Multiple Access (CDMA) spreading or space-time codes, or by simply exploiting the multiple paths, partially or fully uncorrelated, between the transmitting paths and the receiver. The multiple replicas are then appropriately combined at the receiver, resulting in the same or similar performance as that of receive diversity. Thus, in cases when it is practically difficult to employ multiple antennas at the receiver, the beneficial effects of diversity can still be achieved; the cost is higher complexity, since this requires channel-state knowledge by the transmitter. The systems of this structure are generally referred in the literature as Multiple-Input Single Output (MISO) systems.

Several works have been published in the past concerning the concept of optimizing transmitter diversity systems. In [4], Cavers presented an optimal power allocation method, aiming at the minimization of the average bit-error-rate (ABER) under power constraints, when the system is operating over independent or correlated Rayleigh fading channels. This work was extended in [5], considering Nakagami- $m$ fading channels, taking into account the effect of imperfect channel estimation at the transmitter; this concept was also employed in [6], where the optimization was held in terms of the outage probability.

In this letter, we propose an algorithmic solution to the dual power allocation problem, which minimizes and optimally allocates the total transmitting power provided that the instan- taneous BER does not exceed a predetermined threshold. In fact, the transmitter utilizes its knowledge on the amplitude of each individual channel between a transmitting antenna and the receiver, in order to activate the minimum number of branches and to save as much energy as possible. The power allocation problem is formulated as a special case of the well-known fractional minimization Knapsack problem [7], and applies to many practical applications where the power transmitted by each antenna is constrained. Its exact algorithmic solution is computed within a relatively short amount of time, ensuring that no extra delay in packet transmission is induced. Moreover, the rate that the proposed power allocation procedure is repeated depends on the channels' fading coherence time, since the channel state in the time interval between two consecutive procedure repetitions needs to remain unchanged.

\section{SySTEM MODEL}

We consider a typical MISO system, consisting of an $L$ antenna transmitter and a single-antenna receiver. The receiver is equipped with a maximal ratio combiner (MRC), along with an appropriate path-resolving mechanism in order to separate the signals incident from different antennas, e.g. a despreading matched filter when the multiple signals are separated using CDMA spreading codes. Additionally, we assume that each antenna is equipped with its own power amplifier (see e.g., [8][9]), and thus the power transmitted by each antenna is constrained, due to the linearity of the corresponding amplifiers. It is also assumed that the channel amplitudes are known at the receiver, and each of the individual channels is considered independent, but not necessarily identically distributed. In the following, the term branch will denote the individual channel between a transmitting antenna and the receiver.

Let $g_{i}, P_{i}$ represent respectively the instantaneous squared channel gain, including the path-loss and fading attenuation, and the normalized transmitting power with respect to 1 $\mathrm{W}$, corresponding to the $i$ th branch with $i=1, \ldots, L$. The maximum power that each antenna can transmit is limited, as it occurs in most practical applications, and is denoted by $P_{\max }$. Then, $P_{i}$ can be expressed as

$$
P_{i}=P_{\max } x_{i},
$$


where $x_{i} \in[0,1]$. Let $N_{0}$ represent the additive white Gaussian noise (AWGN) power and $G_{i}$ be the instantaneous squared channel gain to noise power ratio corresponding to the $i$ th branch, i.e.,

$$
G_{i}=\frac{g_{i}}{N_{0}}
$$

Then, the instantaneous signal to noise ratio (SNR) $\gamma_{i}$ of the $i$ th branch can be expressed as

$$
\gamma_{i}=P_{i} G_{i}
$$

Moreover, the receiver is assumed to have perfect channel state information, so that the SNR at the combiner output, namely $\gamma_{\text {out }}$, can be expressed as

$$
\gamma_{\text {out }}=\sum_{i=1}^{L} \gamma_{i}
$$

\section{Optimal Power Allocation}

\section{A. Preliminaries: The fractional minimization Knapsack prob- lem}

The well-known zero-one minimization Knapsack problem is defined as follows [7]: Given a bound $B$, and a set of $n$ items with profits $p_{j}>0$ and weights $w_{j}>0, j=1, \ldots, n$, select the subset leading to the minimum profit summation provided that the weight summation is not smaller than $B$, e.g.,

$$
\begin{gathered}
\text { minimize } \sum_{j=1}^{n} p_{j} x_{j} \\
\text { subject to } \sum_{j=1}^{n} w_{j} x_{j} \geq B \\
x_{j} \in\{0,1\}, \quad j=1, \ldots, n .
\end{gathered}
$$

The fractional minimization Knapsack problem has a similar definition, with the only difference being that we are able to select a fraction of each item, i.e., $x_{j} \in[0,1], j=1, \ldots, n$.

\section{B. Mode of Operation}

Let us assume that the power allocation procedure is repeated in a rate ensuring constant fading characteristics in each channel during the selection interval; this implies that the selection repetition rate is greater than or equal to $1 / T_{c}$, where $T_{c}$ stands for the channel coherence time. Considering this, the problem of minimizing the total transmitting power provided that the instantaneous BER does not exceed a specified target BER, denoted by $B E R_{t}$, reduces to the problem of minimizing the transmitting power under the constraint that the overall received SNR is greater than or equal to a given

\begin{tabular}{|c|}
\hline Sort the branches in decreasing order of \\
\hline$G_{i}\left(\right.$ e.g., $\left.x_{1}=\max \left[x_{i}\right]\right)$ \\
\hline$j=1 ; x_{i}=0: i=2, \ldots, L$ \\
\hline$\dagger x_{j}=1$ \\
\hline if $\gamma_{\text {out }}>\gamma_{t}$ \\
\hline modify $x_{j}$ such that $\gamma_{\text {out }}=\gamma_{t} ;$ break \\
\hline else \\
\hline if $j \neq L$ \\
\hline$j=j+1$ \\
\hline go to $\dagger$ \\
\hline break \\
\hline
\end{tabular}

Fig. 1. The proposed power allocation algorithm

value $\gamma_{t}$, i.e.,

$$
\begin{gathered}
\text { minimize } \sum_{i=1}^{L} P_{\max } x_{i} \\
\text { subject to } \sum_{i=1}^{L} G_{i} P_{\max } x_{i} \geq \gamma_{t} \\
x_{i} \in[0,1], \quad i=1, \ldots, L .
\end{gathered}
$$

In a BPSK application, for example, $\gamma_{t}$ is related with $B E R_{t}$ through

$$
\gamma_{t}=\left[\operatorname{erfc}^{-1}\left(2 B E R_{t}\right)\right]^{2}
$$

where $\operatorname{erfc}^{-1}(\cdot)$ is the inverse function of the $\operatorname{erfc}(\cdot)$, which can be efficiently evaluated with desired accuracy, using the approximations given in [10]. Also, note that $\operatorname{erfc}^{-1}(\cdot)$ is a standard built-in function in most of the well-known mathematical software packages, such as MAPLE, MATHEMATICA and MATLAB.

In fact, the problem in (6) represents a special case of the so-called fractional minimization Knapsack problem as it was described above. Its peculiarity lies in the equal profit values $\left(p_{j}\right)$ of each selected item, which correspond to the maximum power $P_{\max }$ that can be transmitted by each antenna. For this reason, the exact optimal solution to (6) coincides with the one provided via the Greedy-Split algorithm [7], a computerimplemented description of which is given in Fig. 1. More specifically, the transmitter first sorts the available branches in decreasing order of $G_{i}$; following this order, it allocates full power to each branch successively, until the SNR of the combiner output exceeds $\gamma_{t}$. Then, the power allocated to the latterly accessed branch is modified so as $\gamma_{\text {out }}$ meets the desired level. Consequently, the above policy allocates full power to the "best" branches, and a part of the full power to a single branch in order to achieve precisely the desired BER, if this is possible. All the other branches remain inactive as long as their fading conditions are relatively "bad".

Nevertheless, it must be noted that possibly the desired $B E R_{t}$ cannot be achieved in each repetition of the proposed algorithm. In such case, $P_{\max }$ is allocated to all the available antennas and $\gamma_{\text {out }}$ is still below $\gamma_{t}$. Then, similarly to the 
typical cellular systems, the system administrator can determine the certain number of successive failures of achieving $B E R_{t}$ that lead to an outage. Hence, the outage probability defined above is identical with the outage probability of the conventional MRC diversity scheme, when the SNR threshold equals $\gamma_{t}$. An extensive study of this probability for Nakagami$m$ fading channels can be found in [11]-[12].

\section{Power Efficiency of the Proposed Scheme}

\section{A. Average Total Transmitted Power in independent and iden-} tically distributed (i.i.d.) Rayleigh Fading for $L=2$

Next, a closed-form expression for the average total transmitted power for the special case of two transmitting antennas, when operating over i.i.d Rayleigh fading channels, is derived. Suppose that $G_{1}^{\prime} \geq G_{2}^{\prime}$ represent the order statistics of the instantaneous channel gains to noise power ratios $G_{1}, G_{2}$, i.e.

$$
G_{1}^{\prime}=\max \left(G_{1}, G_{2}\right) \text { and } G_{2}^{\prime}=\min \left(G_{1}, G_{2}\right) .
$$

Denoting as $f_{G}(\cdot)$ the probability density function (PDF), and as $F_{G}(\cdot)$ the cumulative density function (CDF) of the random variables (RVs) $G_{1}$ and $G_{2}$, the PDF of $G_{1}^{\prime}$ can be expressed as [13, eq. (6-55), (6-58)]

$$
f_{G_{1}^{\prime}}\left(G_{1}^{\prime}\right)=2 f_{G}\left(G_{1}^{\prime}\right) F_{G}\left(G_{1}^{\prime}\right)
$$

The average total transmitted power, namely $P_{a v}$, can be evaluated by taking into account the following two cases:

- when $P_{\max } G_{1}^{\prime} \geq \gamma_{t}$, only a single antenna transmits at a power level which is adjusted so as the SNR meets the threshold $\gamma_{t}$

- when $P_{\max } G_{1}^{\prime}<\gamma_{t}$, the antenna corresponding to the best channel transmits at full power while the power allocated to the remaining antenna is the power required to attain $\gamma_{t}$; if $\gamma_{t}$ cannot be achieved, then this power equals to $P_{\text {max }}$.

Considering the above, $P_{a v}$ can be written as

$$
\begin{aligned}
P_{a v}= & P_{\max } E\left[x_{1}+x_{2}\right]= \\
& P_{\max }\left[\left(1+E\left[\min \left(1, \frac{A-G_{1}^{\prime}}{G_{2}^{\prime}}\right) \mid G_{1}^{\prime}<A\right]\right)\right. \\
& \left.\operatorname{Pr}\left\{G_{1}^{\prime}<A\right\}+E\left[\frac{A}{G_{1}^{\prime}} \mid G_{1}^{\prime} \geq A\right] \operatorname{Pr}\left\{G_{1}^{\prime} \geq A\right\}\right] \\
= & P_{\max }\left(\int_{0}^{A} f_{G_{1}^{\prime}}\left(G_{1}^{\prime}\right) d G_{1}^{\prime}\right. \\
& +\int_{0}^{A} \int_{0}^{G_{1}^{\prime}} \Theta\left(G_{1}^{\prime}, G_{2}^{\prime}\right) f_{G_{1}^{\prime} G_{2}^{\prime}}\left(G_{1}^{\prime}, G_{2}^{\prime}\right) d G_{2}^{\prime} d G_{1}^{\prime} \\
& \left.+\int_{A}^{\infty} \frac{A}{G_{1}^{\prime}} f_{G_{1}^{\prime}}\left(G_{1}^{\prime}\right) d G_{1}^{\prime}\right),
\end{aligned}
$$

where $f_{G_{1}^{\prime} G_{2}^{\prime}}(\cdot, \cdot)$ represents the joint PDF of $G_{1}^{\prime}$ and $G_{2}^{\prime}$, $E[\cdot]$ denotes expectation, $E[B \mid \mathcal{B}]$ stands for the expectation value of $B$ conditioned on the random event $\mathcal{B}, A=\gamma_{t} / P_{\max }$ and

$$
\Theta\left(G_{1}^{\prime}, G_{2}^{\prime}\right)=\left\{\begin{array}{cc}
1, & G_{2}^{\prime} \leq A-G_{1}^{\prime} \\
\frac{A-G_{1}^{\prime}}{G_{2}^{\prime}}, & G_{2}^{\prime}>A-G_{1}^{\prime}
\end{array} .\right.
$$

Therefore, from (11) and simplifying $f_{G_{1}^{\prime} G_{2}^{\prime}}(\cdot, \cdot)$ according to the order statistics theory [14], (10) yields

$$
\begin{aligned}
P_{a v}= & P_{\max }\left(\int_{0}^{A} f_{G_{1}^{\prime}}\left(G_{1}^{\prime}\right) d G_{1}^{\prime}\right. \\
& +2 ! \int_{0}^{\frac{A}{2}} \int_{0}^{G_{1}^{\prime}} f_{G}\left(G_{1}^{\prime}\right) f_{G}\left(G_{2}^{\prime}\right) d G_{2}^{\prime} d G_{1}^{\prime} \\
& +2 ! \int_{\frac{A}{2}}^{A} \int_{0}^{A-G_{1}^{\prime}} f_{G}\left(G_{1}^{\prime}\right) f_{G}\left(G_{2}^{\prime}\right) d G_{2}^{\prime} d G_{1}^{\prime} \\
& +2 ! \int_{\frac{A}{2}}^{A} \int_{A-G_{1}^{\prime}}^{G_{1}^{\prime}} \frac{A-G_{1}^{\prime}}{G_{2}^{\prime}} f_{G}\left(G_{1}^{\prime}\right) f_{G}\left(G_{2}^{\prime}\right) d G_{2}^{\prime} d G_{1}^{\prime} \\
& \left.+\int_{A}^{\infty} \frac{A}{G_{1}^{\prime}} f_{G_{1}^{\prime}}\left(G_{1}^{\prime}\right) d G_{1}^{\prime}\right) \\
= & P_{\max }\left(\mathcal{I}_{1}+\mathcal{I}_{2}+\mathcal{I}_{3}+\mathcal{I}_{4}+\mathcal{I}_{5}\right) .
\end{aligned}
$$

If $G_{1}$ and $G_{2}$ i.i.d exponential RVs with scale parameter $1 / \bar{G}$, using trivial integral simplifications and the non-trivial one given in [15, eq. (2.325)], the integrals in (12) can be written as

$$
\begin{gathered}
\mathcal{I}_{1}=e^{-\frac{2 A}{\bar{G}}}\left(e^{\frac{A}{G}}-1\right)^{2} \\
\mathcal{I}_{2}=2 e^{-\frac{A}{\bar{G}}}\left(e^{\frac{A}{2 \bar{G}}}-\frac{A}{2 \bar{G}}-1\right) \\
\mathcal{I}_{3}=e^{-\frac{A}{\bar{G}}}\left(e^{\frac{A}{2 \bar{G}}}-1\right)^{2} \\
\mathcal{I}_{4}=e^{-\frac{A}{\bar{G}}}\left(e^{-\frac{A}{G}}-2 \mathcal{E}+\frac{A}{\bar{G}}-2 e^{\frac{A}{\bar{G}}}\left(1-\frac{A}{\bar{G}}\right)\right. \\
\times \operatorname{Ei}\left[-\frac{2 A}{\bar{G}}\right]+2\left(1+e^{\frac{A}{\bar{G}}}-\frac{A e^{\frac{A}{G}}}{\bar{G}}\right) \\
\left.\times \operatorname{Ei}\left[-\frac{A}{\bar{G}}\right]+\ln (4)-2 \ln \left(\frac{A}{\bar{G}}\right)-1\right) \\
\mathcal{I}_{5}=\frac{2 A}{\bar{G}}\left(\operatorname{Ei}\left[-\frac{2 A}{\bar{G}}\right]-\operatorname{Ei}\left[-\frac{A}{\bar{G}}\right]\right)
\end{gathered}
$$

where $\mathrm{Ei}[\cdot]$ denotes the exponential integral function defined in [15, eq. (8.211)] and $\mathcal{E}$ the Euler's constant with numerical value $\simeq 0.57721$. Hence, the average total transmitted power can be derived in closed form by substituting (13)-(17) into (12).

\section{B. Average Number of Active Antennas}

The power saving of the proposed scheme can be also quantified by the average number of active antennas at the transmitter. By optimally allocating the transmitted power, not all the available antennas are used to achieve the desired BER. However, this average number of used antennas does not 
correspond exactly to the average transmitted power, since not all the antennas transmit equal amount of power.

By following a similar analysis as in [16], where the GSC and MRC with an output threshold were studied, the average number of active antennas at the transmitter in the proposed scheme is given by

$$
N=1+\sum_{i=1}^{L-1} F^{(L / i)}\left(\gamma_{t}\right) .
$$

where $F^{(L / i)}(\cdot)$ is the CDF of the combined output SNR, of a receiver that uses the $i$ strongest branches out of the $L$ available ones. Fortunately, the desired CDF, is the CDF of a GSC receiver that uses the $i$ strongest branches out of the $L$ available ones, and has been extensively studied in the literature (see e.g., [17]-[18]). For the case of i.i.d and independent but not necessarily identically distributed (i.n.i.d.) Nakagami- $m$ fading the desired CDF was given in [17] and [18] respectively, while for the case of i.i.d. Rayleigh fading a compact formula can be found in [16].

\section{Simulations And Numerical Examples}

In this section, we provide simulations and numerical results demonstrating the considerable reduction in transmitting power that the proposed model offers, compared to the classic equi-power transmitter diversity system, where all the available branches are considered to transmit equal power so that the total transmitted power is the minimum required to achieve a predetermined ABER, if possible. The simulation was conducted on the system model described in Section II, employing BPSK modulation with three or five transmitting antennas and operating over i.i.d. or i.n.i.d. Nakagami-m fading environments.

In Fig. 2, we compare the conventional equi-power scheme (CES) with the new proposed one, assuming three and five transmitting antennas (Fig 2 (a) and 2 (b) respectively), and two different i.i.d. Nakagami- $m$ fading environments. In order to provide a fair comparison in terms of the average total transmitted power, we assume certain amount of power transmitted by the CES, such that the ABER is the same as the ABER achieved by the proposed model. Fig. 2 (b) shows that the proposed scheme offers a noticeable reduction in transmitting power, for a given value of $P_{\max } \bar{G}_{i} / \gamma_{t}$, where $\bar{G}_{i}$ denotes the expectation of $G_{i}$, which is identical for all the available branches. Moreover, this power is nearly the same for both fading environments ( $m=1$ and $m=3$ ), under the condition that there is available power at the transmitter. This important characteristic of the system seems to diminish as the number of antennas reduces $(L=3)$, as it is evident from Fig. 2 (a). Although the total transmitted power is considerably reduced, compared to the CES, the algorithm can hardly maintain the transmitted power at a constant level as the fading conditions worsen. This behavior is easily explained, since any reduction in the available paths between the transmitter and the receiver
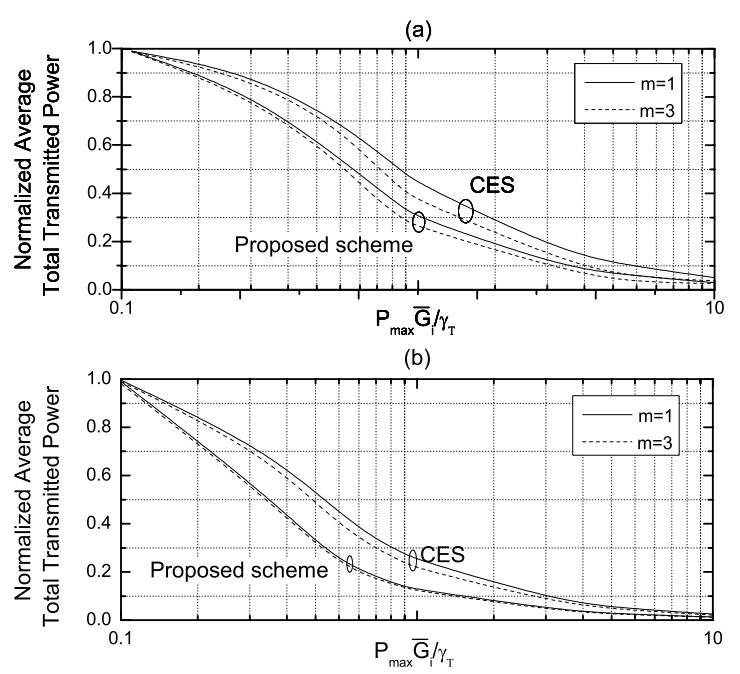

Fig. 2. Average total transmitting power versus $P_{\max } \bar{G}_{i} / \gamma_{t}$, for the conventional equi-power scheme (CES) and the proposed scheme in i.i.d. Nakagami- $m$ fading for (a) $L=3$ and (b) $L=5$.

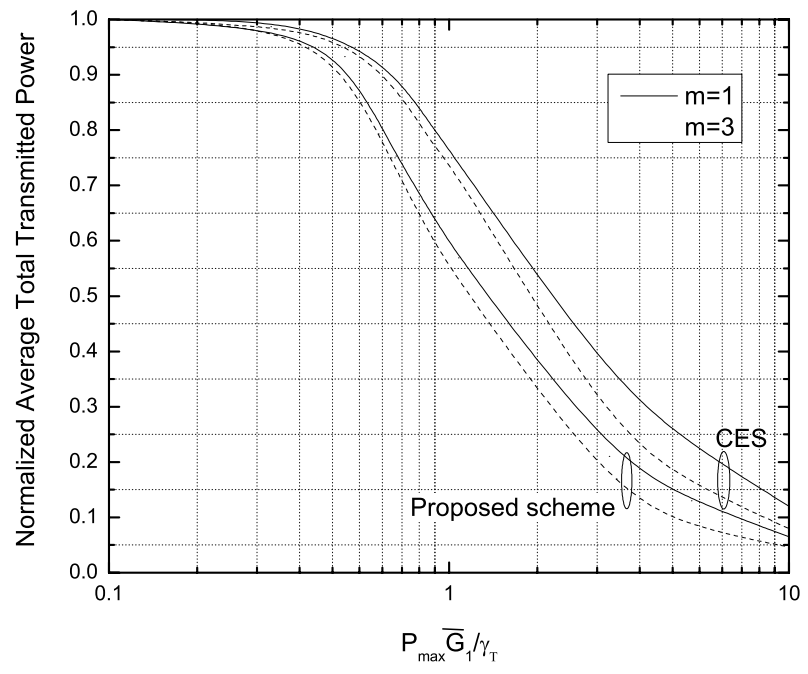

Fig. 3. Average total transmitting power versus $P_{\max } \bar{G}_{1} / \gamma_{t}$, for the conventional equi-power scheme (CES) and the proposed scheme in i.n.i.d. Nakagami- $m$ fading with exponential power profile, for $L=3$.

entails a reduction in the alternative power allocation options among the antennas.

The performance of the proposed power allocation methodology compared to the equivalent CES is depicted in Fig. 3 , when operating over i.n.i.d. Nakagami- $m$ fading channels and $L=3$. In Fig. 3, the average SNRs corresponding to the individual system branches are assumed to follow an exponential profile with unitary power decay factor and initial value denoted by $\bar{G}_{1}$, i.e., $\bar{G}_{i}=\bar{G}_{1} e^{i}$. 


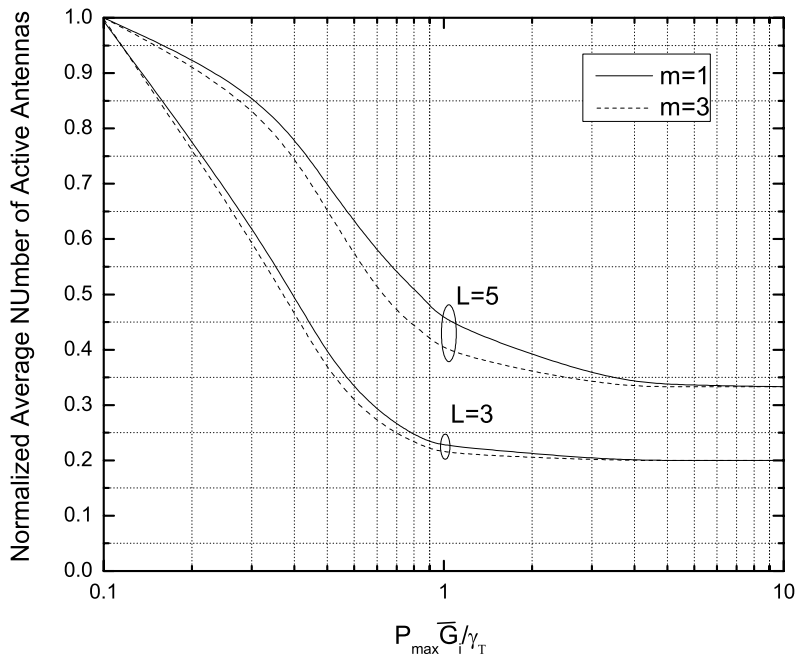

Fig. 4. Average number of active antennas in Nakagami- $m$ fading.

The power saving of the proposed scheme, can be also illustrated through the average number of active antennas at the transmitter. In Fig. 4, two MISO systems with three and five transmitting antennas respectively are assumed, operating over Nakagami- $m$ fading conditions. As it was expected, the more the available transmitting antennas, the less the average active antennas, since the number of alternative power allocation options increases as $L$ increases as well.

\section{ACKNOWLEDGMENT}

This work was performed within the framework of the Satellite Communications Network of Excellence (SatNEx) project (IST-507052) and its Phase-II, SatNEx-II (IST- 27393), funded by the European Commission (EC) under its FP6 program. It was also funded in part by the General Secretariat for Research and Technology (GSRT) of the Hellenic Ministry of Development under a grant for the Bilateral S\&T Cooperation between the Hellenic Republic and Tunisia.

\section{REFERENCES}

[1] J. H. Winters, "The diversity gain of transmit diversity in wireless systems with Rayleigh fading," IEEE Trans. Veh. Technol., vol. 47, pp. 119-123, Feb. 1998.

[2] P. A. Dighe, R. K. Mallik, and S. S. Jamuar, "Analysis of K-transmit dual-receive diversity with cochannel interferers over a Rayleigh fading channel," Wireless Personal Communications, vol. 25, pp. 87-100, May 2003.

[3] _ - "Analysis of transmit-receive diversity in Rayleigh fading," IEEE Trans. Commun., vol. 51, pp. 694-703, Apr. 2003.

[4] J. K. Cavers, "Optimized use of diversity modes in transmitter diversity systems," in Proc. of IEEE Vehicular Technology Conf. VTC'99, Apr. 1999 , pp. $1768-1773$

[5] Y.-Ko and M.-S. Alouini, "Estimation of Nakagami- $m$ fading channel parameters with application to optimized transmitter diversity systems," IEEE Trans. Wir. Commun., vol. 2, pp. 250-259, Mar. 2003.

[6] Y. Xie, C. N. Georghiades, and A. Arapostathis, "Minimum outage probability transmission with imperfect feedback for MISO fading channels," IEEE Trans. Wireless Commun., vol. 4, pp. 1084-1091, May 2005.

[7] H. Kellerer, U. Pferschy, and D. Pisinger, Knapsack Problems, 1st ed. Berlin: Springer, 2004.

[8] W. Yu, "Uplink - downlink duality via minimax duality," IEEE Trans. Inform. Theory, vol. 52, pp. 361-374, Feb. 2006.

[9] W. Yu and T. Lan, "Transmitter optimization for the multi-antenna downlink with per-antenna power constraints," IEEE Trans. Signal Processing, to appear.

[10] Wolfram. (2006) The Wolfram functions site. Internet. [Online]. Available: http://functions.wolfram.com/06.31.06.0001.01

[11] M.-S. Alouini, A. Abdi, and M. Kaveh, "Sum of gamma variates and performance of wireless communication systems over Nakagami-fading channels," IEEE Trans. Veh. Technol., vol. 50, pp. 1471-1480, Nov. 2001.

[12] G. K. Karagiannidis, N. C. Sagias, and T. A. Tsiftsis, "Closed-form statistics for the sum of squared Nakagami- $m$ variates and its applications," IEEE Trans. Commun., to appear.

[13] A. Papoulis, Probability, Random Variables, and Stochastic Procceses, 3rd ed. McGraw-Hill, 1991.

[14] P. J. Bickel and K. Doksum, Mathematical Statistics: Basic Ideas and Selected Topics, 1st ed. Oakland, CA: Holden-Day, 1977.

[15] I. S. Gradshteyn and I. M. Ryzhik, Table of Integrals, Series, and Products, 6th ed. New York: Academic, 2000.

[16] H.-C. Yang and M.-S. Alouini, "MRC and GSC Diversity Combining With an Output Threshold," IEEE Trans. Veh. Technol., vol. 54, no. 3, pp. 1081-1090, May 2005.

[17] A. Annamalai and C. Tellambura, "A new approach to performance evaluation of generalized selection diversity receivers in wireless channels," IEEE Vehicular Technology Conf., vol. 4, pp. 2309-2313, Oct. 2001.

[18] A. Annamalai, G. K. Deora, and C. Tellambura, "Theoretical Diversity Improvement in GSC(N, L) Receiver With Nonidentical Fading Statistics," IEEE Trans. Commun., vol. 53, no. 6, pp. 1027-1035, June 2005. 